\title{
A micromechanics-inspired constitutive model for shape-memory alloys
}

\author{
Amir Sadjadpour ${ }^{1}$ and Kaushik Bhattacharya \\ Division of Engineering and Applied Science, California Institute of Technology, Pasadena, \\ CA 91125 , USA \\ E-mail: amirs@caltech.edu
}

Received 18 March 2007, in final form 16 July 2007

Published 22 August 2007

Online at stacks.iop.org/SMS/16/1751

\begin{abstract}
This paper presents a three-dimensional constitutive model for shape-memory alloys that generalizes the one-dimensional model presented earlier (Sadjadpour and Bhattacharya 2007 Smart Mater. Struct. 16 S51-62). These models build on recent micromechanical studies of the underlying microstructure of shape-memory alloys, and a key idea is that of an effective transformation strain of the martensitic microstructure. This paper explains the thermodynamic setting of the model, demonstrates it through examples involving proportional and non-proportional loading, and shows that the model can be fitted to incorporate the effect of texture in polycrystalline shape-memory alloys.
\end{abstract}

\section{Introduction}

Shape-memory alloys exhibit strongly nonlinear thermomechanical behavior associated with abrupt changes in their lattice structure called martensitic phase transformations. Two common manifestations of this phase transformation are the shape-memory effect wherein an apparently plastic deformation sustained below some critical temperature is recovered on heating above it, and superelasticity wherein significant deformations suffered under loading are recovered on unloading. In this paper, we present a constitutive model for such materials, generalizing our previous work in one dimension [26].

The various applications of shape-memory alloys have motivated a variety of constitutive models, for example $[1,2,6-8,11,13,15,16,18,19,24-29]$ and the references therein. The challenge in modeling these materials is to find a balance between simplicity and an adequate description of the underlying microstructure and its evolution. Our models describe the consequences of microstructure by introducing the effective transformation strain of the martensite. It is the average transformation strain of the different variants of martensite averaged over a representative volume containing multiple grains after the material has formed an allowable microstructure. It is constrained to take values inside a set that is called the set of the effective transformation strains. The micromechanical basis for this set can be found in Bhattacharya and Kohn [4] (also see [5, 22]). Our models also use lessons from

\footnotetext{
1 Author to whom any correspondence should be addressed.
}

micromechanics in introducing kinetic laws that describe the phase transformation and evolution of the effective transformation strain. We refer the reader to [26] for further discussion of the background and setting of our work.

We develop the model in section 2. We introduce the key kinematic concepts, explain the thermodynamic setting and describe the specific constitutive assumptions. As already mentioned, an important kinematic variable is the effective transformation strain that is constrained to lie in a prescribed set. We explain how this set is prescribed and explore the structure of various constitutive prescriptions of this set, especially isotropy and symmetry. We also develop laws governing temperature evolution under various heat transfer conditions. Finally, we discuss the critical stress at which phase transformation begins in this model, and show how the transformation yield surface is the convex dual of the set of effective transformation strains.

We demonstrate the model under the assumption of isotropy in section 3, and without this assumption in section 4. We conduct various parameter studies and show how the parameters can be fitted to experimental data. We demonstrate thermomechanical coupling by studying stressstrain behavior of uniaxial tension, uniaxial compression, pure shear and biaxial tension-compression tests at different initial temperatures.

We demonstrate the response of the model to nonproportional loading by studying an example motivated by combined tension-torsion. Finally we show that our model can 
be fitted to match the anisotropy observed by Inoue et al [10] in rolled sheets.

\section{Constitutive model}

We develop and discuss our model in this section. While this discussion is self-contained, further details of the background behind our definitions as well as some thermodynamic calculations can be found in $[25,26]$.

\subsection{Kinematics}

Consider a typical device made of a shape-memory alloy. It is made up of an extremely large number of grains. In turn, each grain contains a complex microstructure of the austenite phase and the different variants of the martensite phase. Our goal is a macroscopic description. So we take a multiscale point of view and think of each material point of our macroscopic continuum to correspond to a representative volume element (RVE) consisting of a number of grains, each containing a complex microstructure of austenite and variants of martensite. We introduce two kinematic or field or internal variables to represent the consequences of the microstructure in an RVE. The first is the volume fraction of martensite phase, $\lambda(x, t)$. It is a scalar, and is constrained to take values between 0 and 1. The second is the effective transformation strain of the martensite, $\varepsilon_{\mathrm{m}}(x, t)$. It is the average transformation strain of every subregion of martensite in the RVE. It is constrained to lie inside a set $P$, the set of all possible effective transformation strains. We postpone a detailed discussion of this set until section 2.4, but note for now that it is a convex set in the space of symmetric trace-free second-order matrices. Thus

$$
\lambda \in[0,1] \quad \text { and } \quad \varepsilon_{\mathrm{m}} \in P .
$$

It is worth noting that the effective transformation strain of the RVE is $\lambda \varepsilon_{\mathrm{m}}$ since $\lambda$ is the volume fraction of martensite and $\varepsilon_{\mathrm{m}}$ is the effective transformation strain of the martensite. The transformation strain of the austenite is zero by choice of reference configuration.

Finally, we introduce the plastic strain tensor $\varepsilon_{\mathrm{p}}$ as an additional field variable. Putting everything together, we say that the total strain can be divided into three parts, elastic, transformation and plastic:

$$
\begin{aligned}
& \varepsilon(x, t)=\frac{1}{2}\left(\nabla u+\nabla u^{\mathrm{T}}\right) \\
& \quad=\varepsilon_{\mathrm{e}}(x, t)+\lambda(x, t) \varepsilon_{\mathrm{m}}(x, t)+\varepsilon_{\mathrm{p}}(x, t) .
\end{aligned}
$$

\subsection{Balance laws}

We assume that the usual balance laws hold. In local form, the balance of linear momentum and energy may be stated as

$$
\begin{gathered}
\rho u_{t t}=\operatorname{div} \sigma \\
\dot{\epsilon}=-\nabla q+r+\sigma: \dot{\varepsilon}
\end{gathered}
$$

where $\rho$ is the (referential) mass per unit length, $\sigma$ is the (Piola-Kirchhoff) stress, $\epsilon$ is the internal energy density, $q$ the heat flux and $r$ the radiative heating. We also use the local form of the second law of thermodynamics:

$$
-\dot{W}-\eta \dot{\theta}+\sigma: \dot{\varepsilon}-\frac{q \nabla \theta}{\theta} \geqslant 0,
$$

where $W=\epsilon-\theta \eta$ is the Helmholtz free energy density, $\eta$ the entropy density and $\theta$ the (absolute) temperature.
2.3. Constitutive relations, driving forces and kinetic relations

We assume that the Helmholtz free energy density depends on the strain, the temperature and the internal variables:

$$
W=W\left(\varepsilon, \lambda, \varepsilon_{\mathrm{m}}, \varepsilon_{\mathrm{p}}, \theta\right) .
$$

Specifically, we assume

$$
\begin{aligned}
W= & \frac{1}{2}\left(\varepsilon-\varepsilon_{\mathrm{p}}-\lambda \varepsilon_{\mathrm{m}}\right) C\left(\varepsilon-\varepsilon_{\mathrm{p}}-\lambda \varepsilon_{\mathrm{m}}\right) \\
& +\lambda \omega(\theta)-c_{\mathrm{p}} \theta \ln \left(\frac{\theta}{\theta_{0}}\right)
\end{aligned}
$$

where $C$ is a fourth-order tensor denoting the elastic modulus (assumed to be equal in both the austenite and the martensite), $\omega$ is the difference in chemical energy between the austenite and the martensite, $c_{\mathrm{p}}$ is the heat capacity (assumed to be equal in both the austenite and the martensite) and ordinary thermal expansion is neglected. We further assume that

$$
\omega(\theta)=\frac{\mathcal{L}}{\theta_{\text {cr }}}\left(\theta-\theta_{\text {cr }}\right)
$$

where $\mathcal{L}$ is the latent heat of transformation and $\theta_{\mathrm{cr}}$ is the thermodynamic transformation temperature. Arguing as in [26], we obtain

$$
\begin{gathered}
\sigma=C\left(\varepsilon-\varepsilon_{\mathrm{p}}-\lambda \varepsilon_{\mathrm{m}}\right), \\
\eta=\lambda \frac{\mathcal{L}}{\theta_{\mathrm{cr}}}-c_{\mathrm{p}}\left(1+\ln \left(\frac{\theta}{\theta_{0}}\right)\right), \\
d_{\lambda}=\sigma: \varepsilon_{\mathrm{m}}-\omega, \\
d_{\varepsilon_{\mathrm{m}}}=\lambda \sigma \\
d_{\varepsilon_{\mathrm{p}}}=\sigma
\end{gathered}
$$

where $d_{\lambda}, d_{\varepsilon_{\mathrm{m}}}$ and $d_{\varepsilon_{\mathrm{p}}}$ denote the driving forces associated with the rates of change of their conjugate internal variables, $\lambda, \varepsilon_{\mathrm{m}}$ and $\varepsilon_{\mathrm{p}}$, respectively.

The kinetic relation describing the evolution of the martensite volume fraction $\lambda$ is taken to be the following:

$$
\dot{\lambda}= \begin{cases}\dot{\lambda}^{+}\left(1+\left(d_{\lambda}-d_{\lambda}^{+}\right)^{-1}\right)^{-\frac{1}{p}} & d_{\lambda}>d_{\lambda}^{+} \text {and } \lambda<1 \\ \dot{\lambda}^{-}\left(1+\left(d_{\lambda}^{-}-d_{\lambda}\right)^{-1}\right)^{-\frac{1}{p}} & d_{\lambda}<d_{\lambda}^{-} \text {and } \lambda>0 \\ 0 & \text { otherwise }\end{cases}
$$

where $\dot{\lambda}^{ \pm}, d_{\lambda}^{ \pm}, p$ are material parameters. This relation is shown in figure 1 . Note that this law has a stick-slip feature (i.e. it needs a critical driving force before evolution begins and the evolution proceeds in an effectively rate-independent manner) at small rates, but becomes rate-independent at high rates. The motivation for this law is discussed in [26].

We assume the following plasticity relation:

$$
\begin{gathered}
\dot{\varepsilon}_{\mathrm{p}}=K_{\varepsilon_{\mathrm{p}}}\left(d_{\varepsilon_{\mathrm{p}}}, \text { yield surface }\right)=\frac{\dot{d_{\varepsilon_{\mathrm{p}}}}}{H} \\
= \begin{cases}\frac{\dot{\sigma}}{H} & \sigma \in \text { yield surface } \\
0 & \text { otherwise }\end{cases}
\end{gathered}
$$

where $H$ is the hardening parameter.

The evolution of the effective transformation strain $\varepsilon_{\mathrm{m}}$ describes the twinning, detwinning and other such processes 


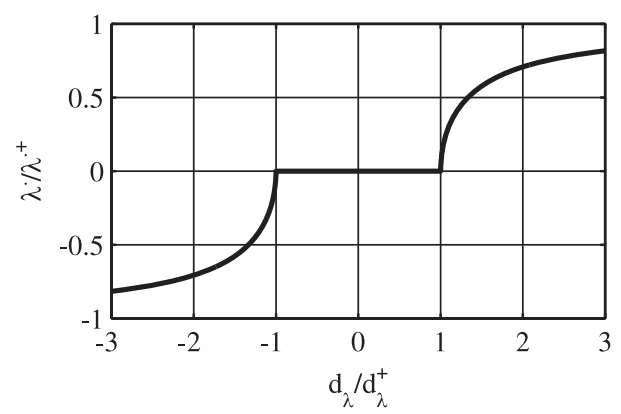

Figure 1. The kinetic relation between $\dot{\lambda}$ and the driving force $d_{\lambda}$.

that convert one martensitic variant to another. We assume a rather simple law for its evolution:

$$
\dot{\varepsilon}_{\mathrm{m}}=K_{\varepsilon_{\mathrm{m}}}\left(d_{\varepsilon_{\mathrm{m}}}, \lambda, \varepsilon_{\mathrm{m}}\right)= \begin{cases}\frac{\alpha}{\lambda} d_{\varepsilon_{\mathrm{m}}} & \varepsilon_{\mathrm{m}} \in \stackrel{\circ}{P} \\ \frac{\alpha}{\lambda}\left(d_{\varepsilon_{\mathrm{m}}}\right)_{\partial P} & \varepsilon_{\mathrm{m}} \in \partial P \\ 0 & \text { otherwise }\end{cases}
$$

where $\stackrel{\circ}{P}$ denotes the interior of $P, \partial P$ the boundary of $P$ and $A_{\partial P}$ the projection of $A$ to the tangent space of $P$. There are subtleties associated with this statement which we presently discuss.

\subsection{Set of effective transformation strains}

In general, the transformation strain $\varepsilon_{\mathrm{m}}$ is a symmetric tensor and thus has six independent components. It may appear therefore that we need a six-dimensional transformation strain space to represent the set of effective transformation strains. Self-accommodation, however, dictates that the transformation strain $\varepsilon_{\mathrm{m}}$ tensor is trace-free [3]. It follows then that the set $P$ lives on a subspace five-dimensional space of trace-free symmetric matrices or deviatoric strains. Therefore, viewed as a six-dimensional object, $\stackrel{\circ}{P}$, the interior of $P$, is empty. However, it is convenient to restrict ourselves to the fivedimensional space of deviatoric strains and rewrite (16) as

$$
\dot{\varepsilon}_{\mathrm{m}}=K_{\varepsilon_{\mathrm{m}}}\left(d_{\varepsilon_{\mathrm{m}}}, \lambda, \varepsilon_{\mathrm{m}}\right)= \begin{cases}\frac{\alpha}{\lambda} \hat{d}_{\varepsilon_{\mathrm{m}}} & \varepsilon_{\mathrm{m}} \in \stackrel{\circ}{ } \\ \frac{\alpha}{\lambda}\left(\hat{d}_{\varepsilon_{\mathrm{m}}}\right)_{\partial P} & \varepsilon_{\mathrm{m}} \in \partial P \\ 0 & \text { otherwise }\end{cases}
$$

where $\stackrel{\circ}{P}$ and $\partial P$ are interpreted as the relative interior and relative boundary to this subspace, and $\hat{A}$ is the deviatoric part of the $A$.

To elaborate on this point, suppose we define $P$ through the following relation:

$$
P=\left\{\varepsilon_{\mathrm{m}} \mid \operatorname{tr}\left(\varepsilon_{\mathrm{m}}\right)=0, g\left(\varepsilon_{\mathrm{m}}\right) \leqslant 0\right\}
$$

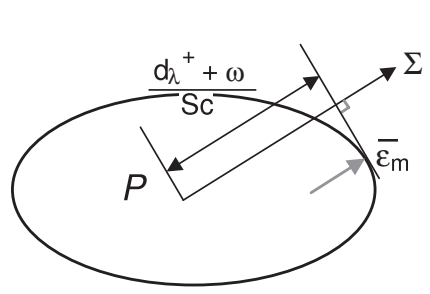

Strain Space
Stress Space

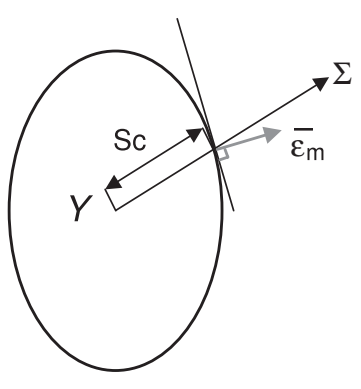

Figure 2. Initiation of the forward martensitic phase transformation.

for a suitable $g: \mathbb{R}_{\mathrm{dev}}^{3 \times 3} \rightarrow \mathbb{R}$. Then

$$
\dot{\varepsilon}_{\mathrm{m}}= \begin{cases}\frac{\alpha}{\lambda} \hat{d}_{\varepsilon_{\mathrm{m}}} & g\left(\varepsilon_{\mathrm{m}}\right)<0 \\ \frac{\alpha}{\lambda}\left(\hat{d}_{\varepsilon_{\mathrm{m}}}-\hat{d}_{\varepsilon_{\mathrm{m}}}: \frac{\partial g}{\partial \varepsilon_{\mathrm{m}}} \frac{\frac{\partial g}{\partial \varepsilon_{\mathrm{m}}}}{\left|\frac{\partial g}{\partial \varepsilon_{\mathrm{m}}}\right|^{2}}\right) & g\left(\varepsilon_{\mathrm{m}}\right)=0 \\ 0 & \text { otherwise. }\end{cases}
$$

Recalling that $d_{\varepsilon_{\mathrm{m}}}=\lambda \sigma$ from (12),

$$
\dot{\varepsilon}_{\mathrm{m}}= \begin{cases}\alpha \hat{\sigma} & g\left(\varepsilon_{\mathrm{m}}\right)<0 \\ \alpha\left(\hat{\sigma}-\hat{\sigma}: \frac{\partial g}{\partial \varepsilon_{\mathrm{m}}} \frac{\frac{\partial g}{\partial \varepsilon_{\mathrm{m}}}}{\left|\frac{\partial g}{\partial \varepsilon_{\mathrm{m}}}\right|^{2}}\right) & g\left(\varepsilon_{\mathrm{m}}\right)=0 \\ 0 & \text { otherwise. }\end{cases}
$$

We now discuss three specific constitutive relations for $P$.

2.4.1. Isotropic and symmetric transformation. Isotropy states that

$$
g\left(\varepsilon_{\mathrm{m}}\right)=g\left(R^{\mathrm{T}} \varepsilon_{\mathrm{m}} R\right)
$$

for all rotations $R$. This implies that we can define the transformation strain function, $g\left(\varepsilon_{\mathrm{m}}\right)$ by three principal values of the transformation strain, or its three invariants $I_{1}\left(\varepsilon_{\mathrm{m}}\right)$, $I_{2}\left(\varepsilon_{\mathrm{m}}\right)$ and $I_{3}\left(\varepsilon_{\mathrm{m}}\right)$ where

$$
\begin{gathered}
I_{1}\left(\varepsilon_{\mathrm{m}}\right)=\operatorname{tr}\left(\varepsilon_{\mathrm{m}}\right), \\
I_{2}\left(\varepsilon_{\mathrm{m}}\right)=\frac{1}{2}\left[\operatorname{tr}\left(\varepsilon_{\mathrm{m}}\right)^{2}-\operatorname{tr}\left(\varepsilon_{\mathrm{m}}^{2}\right)\right], \\
I_{3}\left(\varepsilon_{\mathrm{m}}\right)=\operatorname{det}\left(\varepsilon_{\mathrm{m}}\right) .
\end{gathered}
$$

As mentioned earlier, self-accommodation sets the transformation strain $\varepsilon_{\mathrm{m}}$ to be trace-free, which forces $I_{1}\left(\varepsilon_{\mathrm{m}}\right)$ to vanish, so that the set depends only on the second and third invariants. Further, $I_{2}\left(\varepsilon_{\mathrm{m}}\right)$ simplifies as follows:

$$
I_{2}\left(\varepsilon_{\mathrm{m}}\right)=-\frac{1}{2} \operatorname{tr}\left(\varepsilon_{\mathrm{m}}^{2}\right) .
$$

A very simple constitutive choice for the set of effective transformation strains is

$$
P=\left\{\varepsilon_{\mathrm{m}} \mid \operatorname{tr}\left(\varepsilon_{\mathrm{m}}\right)=0, g\left(\varepsilon_{\mathrm{m}}\right)=I_{2}\left(\varepsilon_{\mathrm{m}}\right)-b \leqslant 0\right\} .
$$

We may regard this as the analog of the von Mises yield criterion in plasticity. Equations (16) and (26) give the growth rule for the transformation strain as 

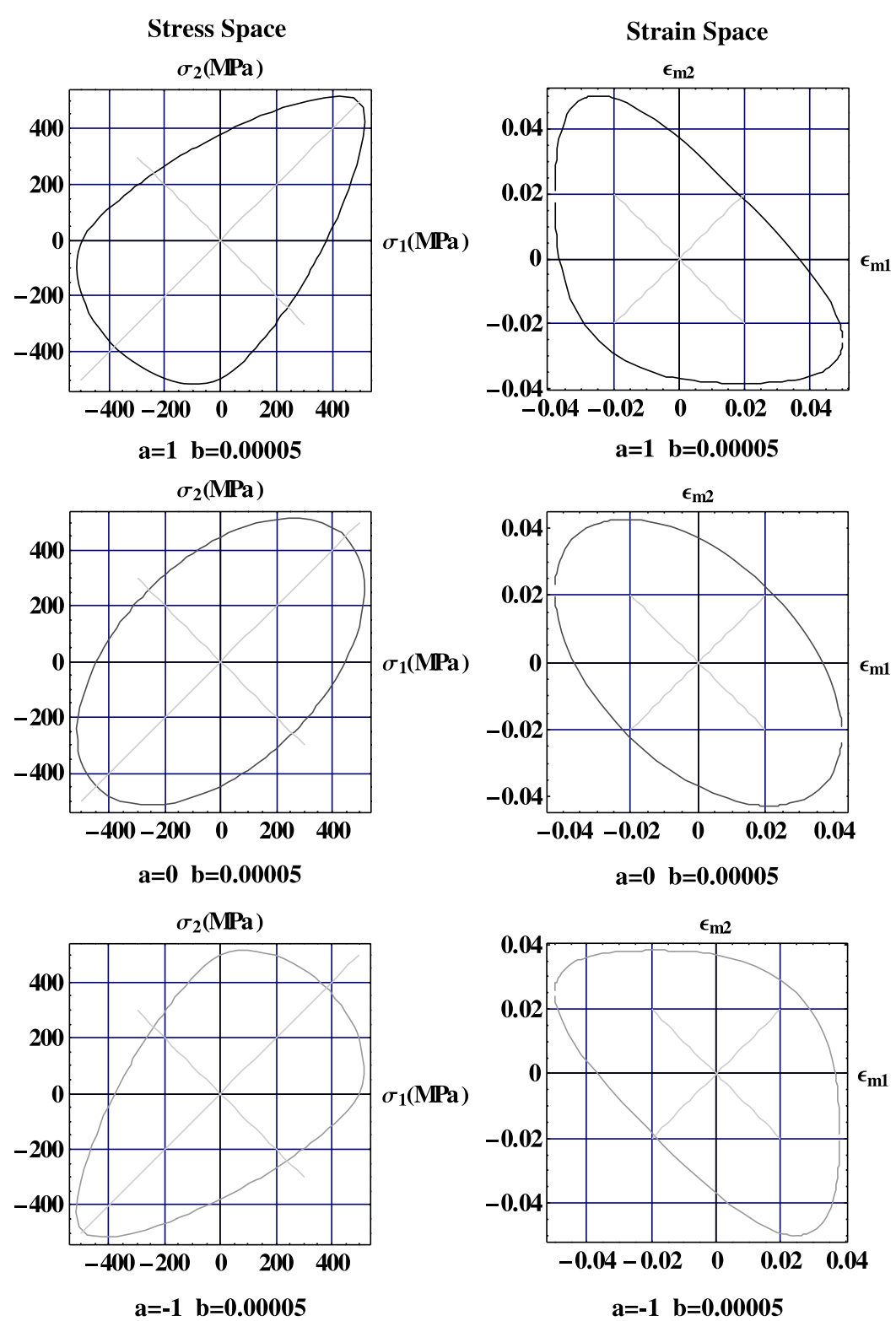

Figure 3. Parameter study on the effect of the parameter $a$ on the shape of the set of effective strain and the transformation yield surface in the isotropic setting.
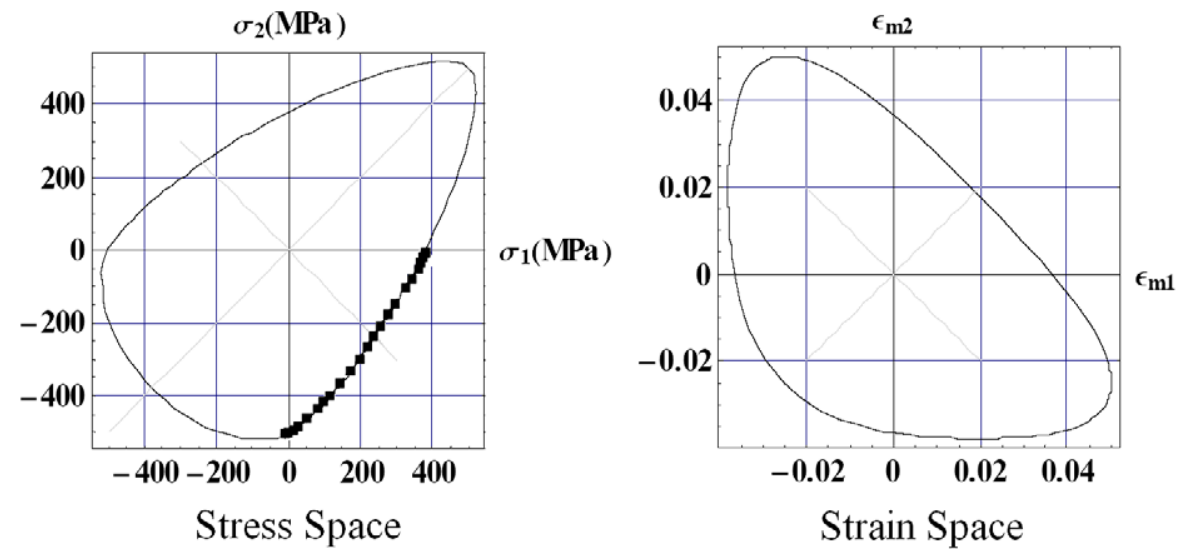

Figure 4. The transformation yield surface and the set of effective transformation strain. The individual marks are experiments of Lexcellent and Blanc [12] while the continuous curve is our fit with the parameters (47). 
A micromechanics-inspired constitutive model for shape-memory alloys
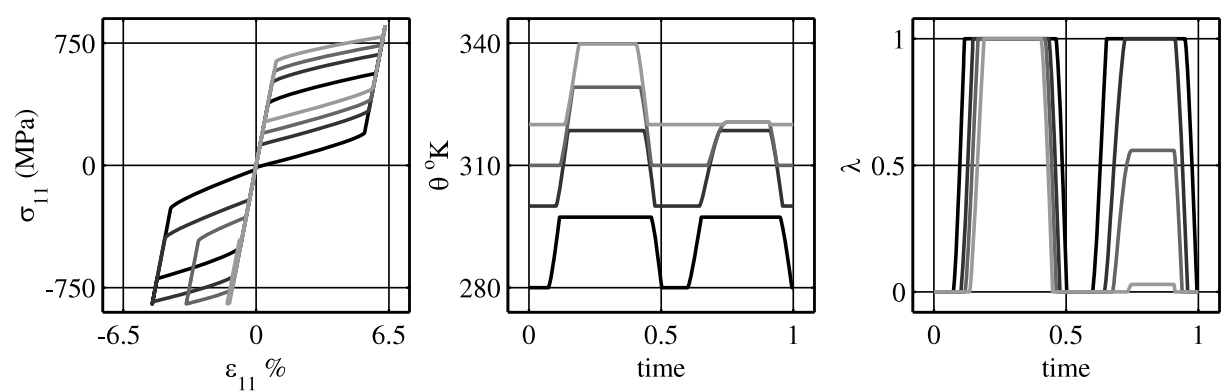

Figure 5. The adiabatic response of a material subjected to a uniaxial tension-compression load with various initial temperatures.
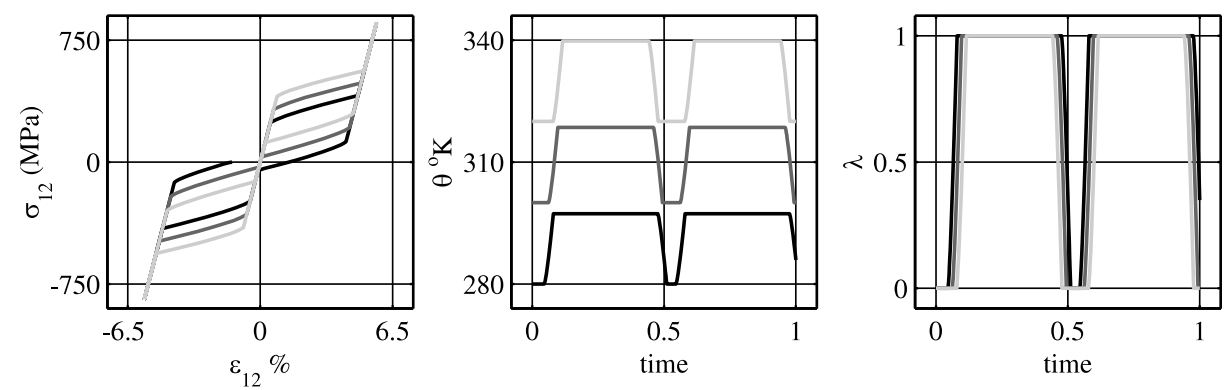

Figure 6. The adiabatic response of a material subjected to a pure shear with various initial temperatures.
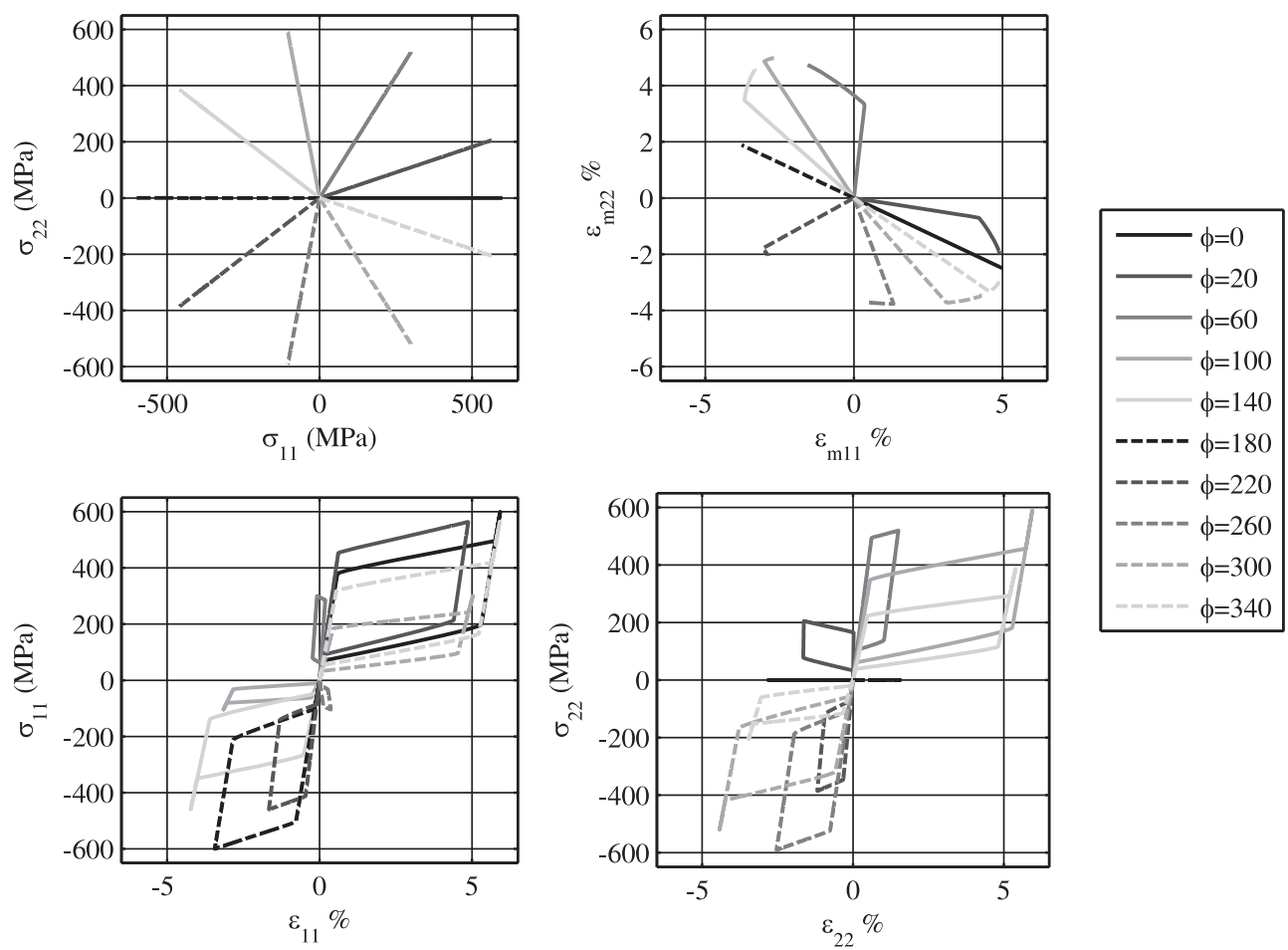

Figure 7. The response of the material subjected to a biaxial proportional tension-compression loading.

$$
\dot{\varepsilon}_{\mathrm{m}}= \begin{cases}\alpha \hat{\sigma} & \left|\varepsilon_{\mathrm{m}}\right|<\sqrt{2 b} \\ \alpha\left(\hat{\sigma}-\varepsilon_{\mathrm{m}} \frac{\varepsilon_{\mathrm{m}}: \hat{\sigma}}{\left|\varepsilon_{\mathrm{m}}\right|^{2}}\right) & \left|\varepsilon_{\mathrm{m}}\right|=\sqrt{2 b} \\ 0 & \text { otherwise. }\end{cases}
$$

Since $g\left(\varepsilon_{\mathrm{m}}\right)=g\left(-\varepsilon_{\mathrm{m}}\right)$, this set displays tension-compression symmetry.
2.4.2. Isotropic and asymmetric transformation. It has long been known (see, for example, Burkart and Read [9]) that shape-memory alloys do not display tensioncompression symmetry. Since $I_{2}\left(\varepsilon_{\mathrm{m}}\right)$ does not change sign as transformation strain changes sign, any constitutive choice based solely on it will necessarily display tension-compression symmetry. Therefore an asymmetric response will have involved $I_{3}\left(\varepsilon_{\mathrm{m}}\right)$. 

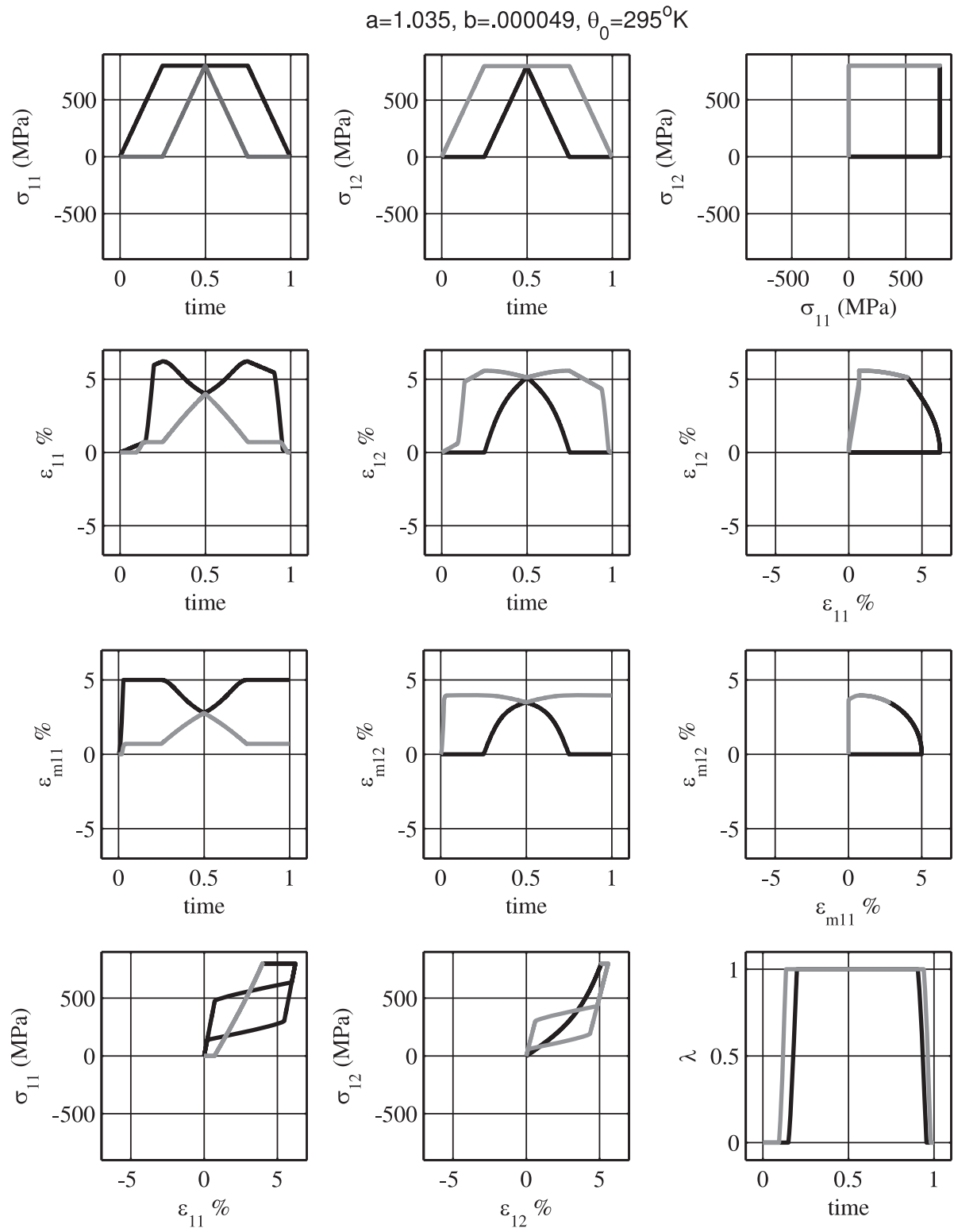

Figure 8. Non-proportional stress-controlled test.

A simple constitutive choice is

$$
g\left(\varepsilon_{\mathrm{m}}\right)=\left(-I_{2}\left(\varepsilon_{\mathrm{m}}\right)\right)^{\frac{3}{2}}-a I_{3}\left(\varepsilon_{\mathrm{m}}\right)-b .
$$

This defines the set of effective transformation strains to be

$$
\begin{aligned}
P & =\left\{\varepsilon_{\mathrm{m}} \mid \operatorname{tr}\left(\varepsilon_{\mathrm{m}}\right)=0, g\left(\varepsilon_{\mathrm{m}}\right)\right. \\
& \left.=\left(-I_{2}\left(\varepsilon_{\mathrm{m}}\right)\right)^{\frac{3}{2}}-a I_{3}\left(\varepsilon_{\mathrm{m}}\right)-b \leqslant 0\right\} .
\end{aligned}
$$

Equations (16) and (29) give the growth rule for the transformation strain as

$$
\dot{\varepsilon}_{\mathrm{m}}= \begin{cases}\alpha \hat{\sigma} & g\left(\varepsilon_{\mathrm{m}}\right)<0 \\ \alpha\left(\hat{\sigma}-\left(\frac{3\left|\varepsilon_{\mathrm{m}}\right|}{\sqrt{8}} \varepsilon_{\mathrm{m}}-a \operatorname{cof}\left(\varepsilon_{\mathrm{m}}\right)^{\mathrm{T}}\right)\right. & \\ \left.\quad \times \frac{\left(\frac{3\left|\varepsilon_{\mathrm{m}}\right|}{\sqrt{8}} \varepsilon_{\mathrm{m}}-a \operatorname{cof}\left(\varepsilon_{\mathrm{m}}\right)^{\mathrm{T}}\right): \hat{\sigma}}{\left|\left(\frac{3\left|\varepsilon_{\mathrm{m}}\right|}{\sqrt{8}} \varepsilon_{\mathrm{m}}-a \operatorname{cof}\left(\varepsilon_{\mathrm{m}}\right)^{\mathrm{T}}\right)\right|^{2}}\right) & g\left(\varepsilon_{\mathrm{m}}\right)=0 \\ 0 & \text { otherwise. }\end{cases}
$$

2.4.3. Transversely isotropic and asymmetric transformation. Shape-memory alloys are often made as wires and tubes by drawing, and as sheets by rolling. These endow the material with a crystallographic texture, i.e. the grains of the polycrystalline specimen are no longer randomly oriented but show a preferential distribution. In such circumstances, the material is no longer isotropic and the function $g$ no longer satisfies (21).

It is common to have a uniaxial texture where one crystallographic axis is preferentially oriented along the drawing or rolling direction. So this direction is special while all other directions normal to it are equivalent. Then,

$g\left(\varepsilon_{\mathrm{m}}\right)=g\left(R^{\mathrm{T}} \varepsilon_{\mathrm{m}} R\right)$

$$
\text { for all rotations } R \text { that satisfy } R \hat{e}=\hat{e} \text {. }
$$

It follows that

$$
g\left(\varepsilon_{\mathrm{m}}\right)=g\left(I_{2}\left(\varepsilon_{\mathrm{m}}\right), I_{3}\left(\varepsilon_{\mathrm{m}}\right), \hat{e} \cdot \varepsilon_{\mathrm{m}} \hat{e}, \hat{e} \cdot \varepsilon_{\mathrm{m}}^{2} \hat{e}\right) .
$$



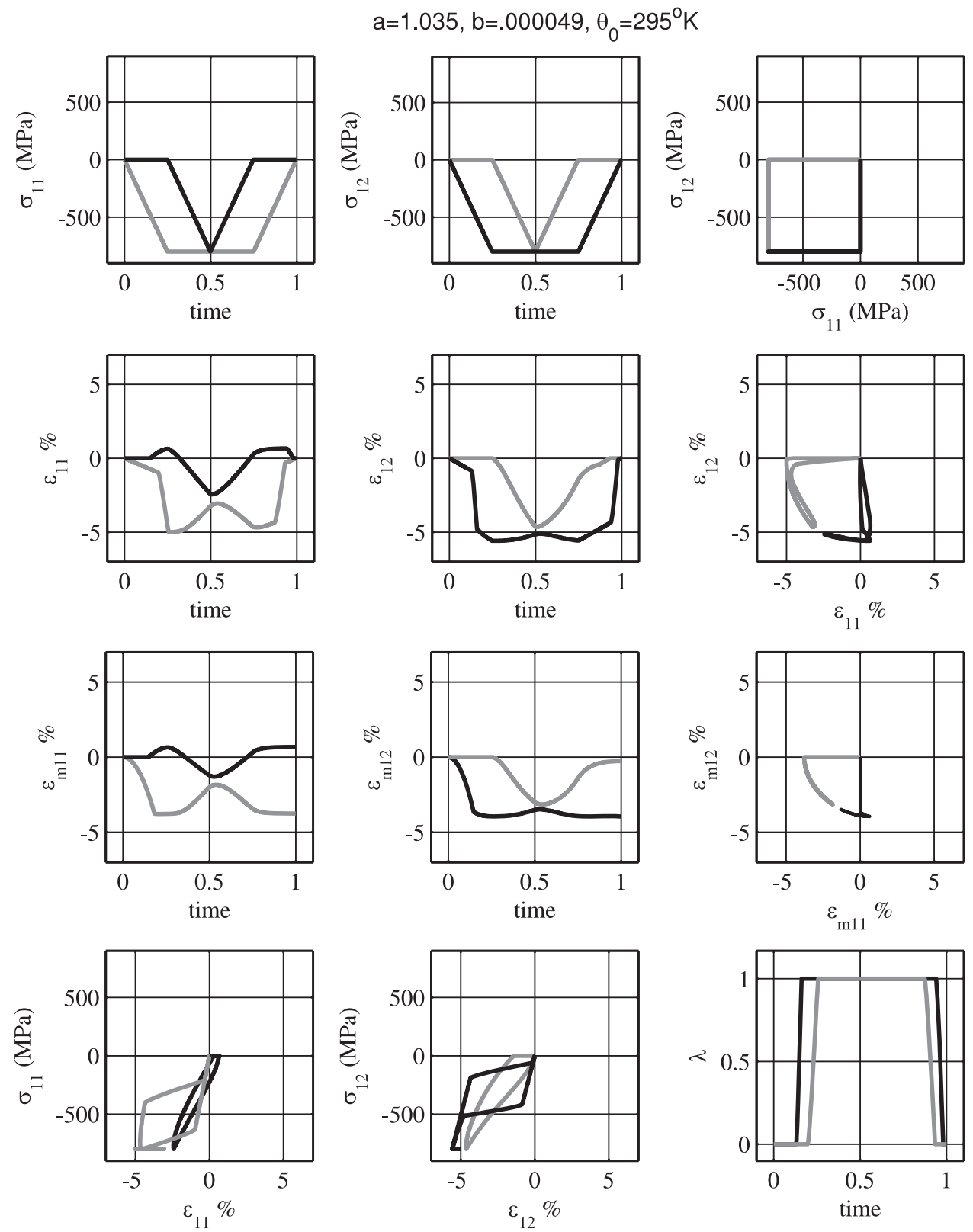

Figure 9. Non-proportional stress-controlled test.

A simple, but useful, choice is

$$
g\left(\varepsilon_{\mathrm{m}}\right)=\left(-I_{2}\left(\varepsilon_{\mathrm{m}}\right)\right)^{\frac{3}{2}}-a I_{3}\left(\varepsilon_{\mathrm{m}}\right)-b-c\left(\hat{e} \cdot \varepsilon_{\mathrm{m}} \hat{e}\right)^{3} .
$$

This defines the set of effective transformation strains to be

$$
\begin{aligned}
P & =\left\{\varepsilon_{\mathrm{m}} \mid \operatorname{tr}\left(\varepsilon_{\mathrm{m}}\right)=0, g\left(\varepsilon_{\mathrm{m}}\right)\right. \\
& \left.=\left(-I_{2}\left(\varepsilon_{\mathrm{m}}\right)\right)^{\frac{3}{2}}-a I_{3}\left(\varepsilon_{\mathrm{m}}\right)-b-c\left(\hat{e} \cdot \varepsilon_{\mathrm{m}} \hat{e}\right)^{3}\right\} .
\end{aligned}
$$

This in turn changes the growth rule for the transformation strain (30) to

$$
\dot{\varepsilon}_{\mathrm{m}}= \begin{cases}\frac{\alpha}{\lambda} \hat{d}_{\varepsilon_{\mathrm{m}}} & g\left(\varepsilon_{\mathrm{m}}\right)<0 \\ \frac{\alpha}{\lambda}\left(\hat{d}_{\varepsilon_{\mathrm{m}}}-\frac{\hat{d}_{\varepsilon_{\mathrm{m}}}: \frac{\partial g}{\partial \varepsilon_{\mathrm{m}}}}{\left|\frac{\partial g}{\partial \varepsilon_{\mathrm{m}}}\right|^{2}}\right) & g\left(\varepsilon_{\mathrm{m}}\right)=0 \\ 0 & \text { otherwise }\end{cases}
$$

where

$$
\frac{\partial g}{\partial \varepsilon_{\mathrm{m}}}=\frac{3\left|\varepsilon_{\mathrm{m}}\right|}{\sqrt{8}} \varepsilon_{\mathrm{m}}-a \operatorname{cof}\left(\varepsilon_{\mathrm{m}}\right)^{\mathrm{T}}-3 c(\hat{e} \cdot \hat{e})\left(\hat{e} \cdot \varepsilon_{\mathrm{m}} \hat{e}\right)^{2} .
$$

We present a detailed parameter study of how $a, b$ and $c$ determine the shape of the set of effective transformation strains in sections 3 and 4 .

\subsection{Temperature evolution}

The energy balance, along with the constitutive relations, describe the evolution of the temperature. Specializing to the specific constitutive relation and in particular (10), we obtain

$$
c_{\mathrm{p}} \dot{\theta}=\dot{\lambda} \theta \frac{\mathcal{L}}{\theta_{\mathrm{cr}}}-\nabla q+r+d_{\lambda} \dot{\lambda}+d_{\varepsilon_{\mathrm{m}}}: \dot{\varepsilon}_{\mathrm{m}}+d_{\varepsilon_{\mathrm{p}}}: \dot{\varepsilon}_{\mathrm{p}}
$$



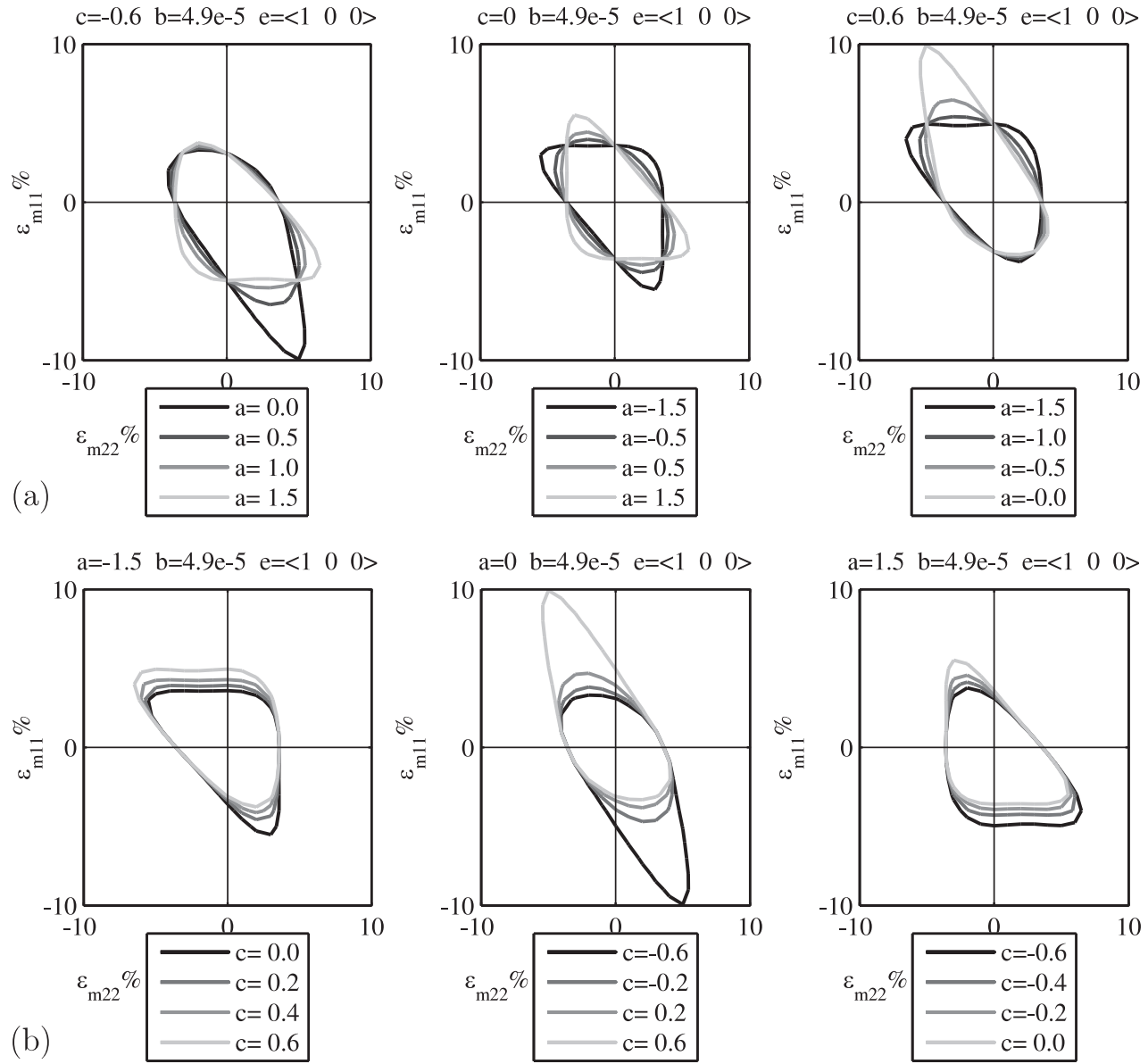

Figure 10. The section of the set of effective transformation strains in the $\varepsilon_{\mathrm{m} 11}-\varepsilon_{\mathrm{m} 22}$ plane for various sets of parameters $a, c$.

For processes where heat transfer is negligible we have $q=r=0$. Further, it turns out that the latent heat of transformation is large compared to the energy dissipated during transformation, martensitic variant reorientation and plasticity during typical processes of interest. Therefore we assume

$$
c_{\mathrm{p}} \dot{\theta}=\theta \dot{\lambda} \frac{\mathcal{L}}{\theta_{\mathrm{cr}}}
$$

Integrating this, we obtain a relation between temperature, volume fraction of martensite, latent heat and specific heat:

$$
\theta(t)=\theta_{0} \exp \left(\frac{\left(\lambda(t)-\lambda_{0}\right) \mathcal{L}}{c_{\mathrm{p}} \theta_{\mathrm{cr}}}\right)
$$

\subsection{Phase transformation yield surface}

Various authors have used a transformation yield surface to characterize shape-memory alloys (see, for example, [12-14]). This surface describes the value of the stress at which a specimen in austenite above the $M_{\mathrm{S}}$ temperature and subjected to a proportional load begins to transform. We now show that this set is closely related to (the convex dual of) our set of transformation strains.

Consider a specimen of shape-memory material in the austenite state $(\lambda=0)$ and above $M_{\mathrm{s}}$ subjected to a proportional load

$$
\sigma=s \Sigma
$$

for fixed $\Sigma$ with $|\Sigma|=1$ and for $s>0$ and monotone increasing. Since we have chosen $\alpha$ to be so large that $\varepsilon_{\mathrm{m}}$ evolves much faster than stress, $\varepsilon_{\mathrm{m}}$ reaches the boundary $\partial P$ of the set of effective transformation strains quickly and then evolves along it till the normal to $\partial P$ becomes parallel to $\Sigma$. This value, $\varepsilon_{\mathrm{m}}=\overline{\varepsilon_{\mathrm{m}}}$, does not evolve any further and solves the following problem:

$$
\max _{\varepsilon_{\mathrm{m}} \in P}\left(\sigma: \varepsilon_{\mathrm{m}}\right)=s \max _{\varepsilon_{\mathrm{m}} \in P}\left(\Sigma: \varepsilon_{\mathrm{m}}\right) .
$$

The driving force for transformation, given by (11), is therefore

$$
d_{\lambda}=\sigma: \overline{\varepsilon_{\mathrm{m}}}-\omega=s \max _{\varepsilon_{\mathrm{m}} \in P}\left(\Sigma: \varepsilon_{\mathrm{m}}\right)-\omega
$$

Transformation begins when $d=d_{\lambda}^{+}$. Thus it follows that the value of the stress at which transformation begins is given by

$$
s_{c}(\Sigma)=\left(d_{\lambda}^{+}+\omega\right) / \max _{\varepsilon_{\mathrm{m}} \in P}\left(\Sigma: \varepsilon_{\mathrm{m}}\right) .
$$

This is demonstrated graphically in figure 2 . We define the set

$$
Y=\left\{\sigma:|\sigma| \leqslant s_{c}(\sigma /|\sigma|)\right\}
$$

to be the yield set and the surface $\partial Y$ to be the transformation yield set. 

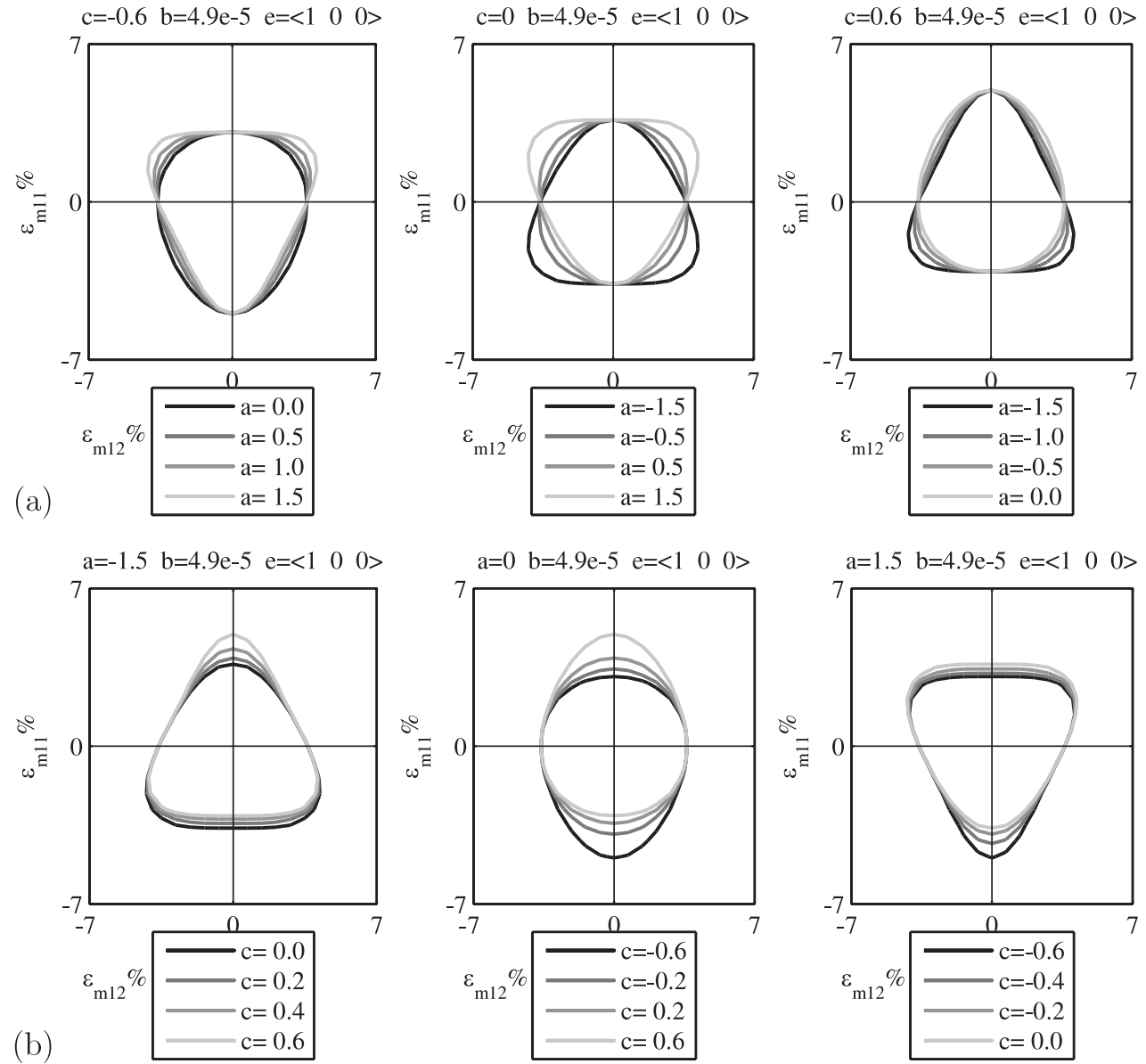

Figure 11. The section of the set of effective transformation strains in the $\varepsilon_{\mathrm{m} 11}-\varepsilon_{\mathrm{m} 12}$ plane for various sets of parameters $a, c$.

\section{Demonstration of the isotropic model}

In this section, we elaborate on and demonstrate our model under the assumption of isotropy.

\subsection{Parameters}

Consistent with typical experiments on $\mathrm{NiTi}$ (see, for example, [17]), we fix the following parameters:

$$
\begin{array}{llrl}
M_{\mathrm{S}}=-51.55^{\circ} \mathrm{C} & \text { and } & & A_{\mathrm{s}}=-6.36^{\circ} \mathrm{C} \\
\mathcal{L}=79\left(\frac{\mathrm{MJ}}{\mathrm{m}^{3}}\right) & \text { and } & c_{\mathrm{p}}=5.4\left(\frac{\mathrm{MJ}}{\mathrm{m}^{3} \mathrm{~K}}\right) \\
E=65(\mathrm{GPa}) & \text { and } & \sigma_{y}=1500(\mathrm{MPa})
\end{array}
$$

where $M_{\mathrm{s}}$ and $A_{\mathrm{s}}$ are the martensite start and austenite start temperatures, respectively. The temperatures are defined in our model through equations (14) and (11) to be

$$
d_{\lambda}^{+}=-\omega\left(M_{\mathrm{s}}\right), \quad d_{\lambda}^{-}=-\omega\left(A_{\mathrm{s}}\right)
$$

\subsection{Set of effective transformation strains}

We consider the set of effective transformation strains defined in (29). This depends on two parameters, $a$ and $b$. It is clear by inspecting (29) that the parameter $b$ simply rescales the set of effective transformation strains, and thus also the transformation yield surface. The parameter $a$, on the other hand, controls the shape of these sets as shown in figure 3 . For each value of $a$, this figure plots the section of the set $P$ in the $\varepsilon_{11}-\varepsilon_{22}$ plane as well as the section of corresponding set $Y$ in the $\sigma_{11}-\sigma_{22}$ plane. Note that the sets are symmetric about the $\varepsilon_{11}=\varepsilon_{22}$ and $\sigma_{11}=\sigma_{22}$ in view of isotropy. The value $a=0$ corresponds to tension-compression symmetry, and both sets are ellipses. Positive values of $a$ allows larger tensile transformation strain, and thus requires smaller tensile stress for transformation. Negative values of $a$ reverse this behavior.

We now fit our model to the experimental observations of Lexcellent and Blanc [12]. They conducted compression pressure experiments on $\mathrm{Ti}-49.75 \mathrm{Ni}$ (at.\%) tubes to obtain the transformation yield surface. We use the critical stress in two directions:

Direction $\langle 100\rangle:\left(\sigma_{1}, \sigma_{2}\right)=(380,0)(\mathrm{MPa})$

$$
\Rightarrow s_{c 1}=380(\mathrm{MPa})
$$

Direction $\langle 1 \overline{1} 0\rangle:\left(\sigma_{1}, \sigma_{2}\right)=(0,-240)(\mathrm{MPa})$

$$
\Rightarrow s_{c 2}=339(\mathrm{MPa})
$$

as well as the measured transformation strain $\varepsilon_{\mathrm{m}}^{\mathrm{t}}=5 \%$ in the $\langle 100\rangle$ direction, to obtain the parameters $a, b$ and $d_{\lambda}^{+}+\omega$ :

$a=1.035, \quad b=0.000049, \quad d_{\lambda}^{+}+\omega=19\left(\frac{\mathrm{MJ}}{\mathrm{m}^{3}}\right)$. 

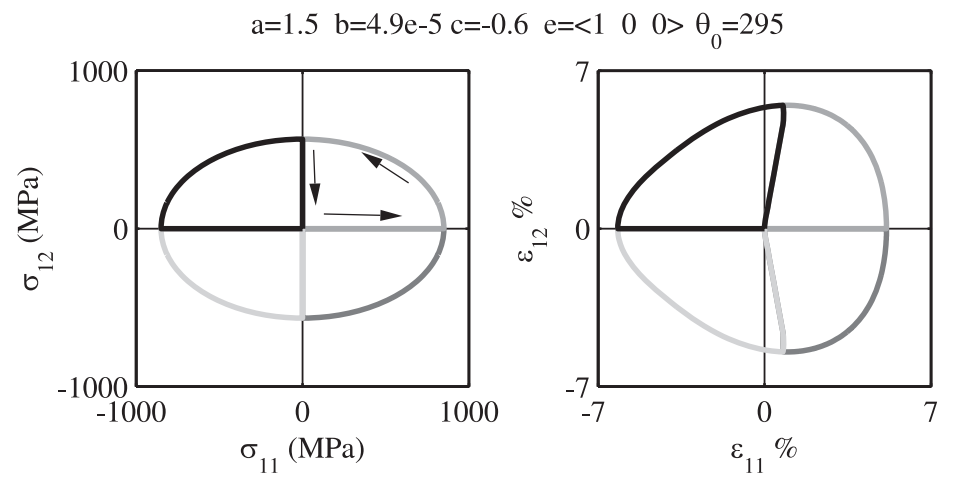

$\mathrm{a}=1.5 \quad \mathrm{~b}=4.9 \mathrm{e}-5 \mathrm{c}=-0.6 \quad \mathrm{e}=<1 \quad 0 \quad 0>\theta_{0}=295$
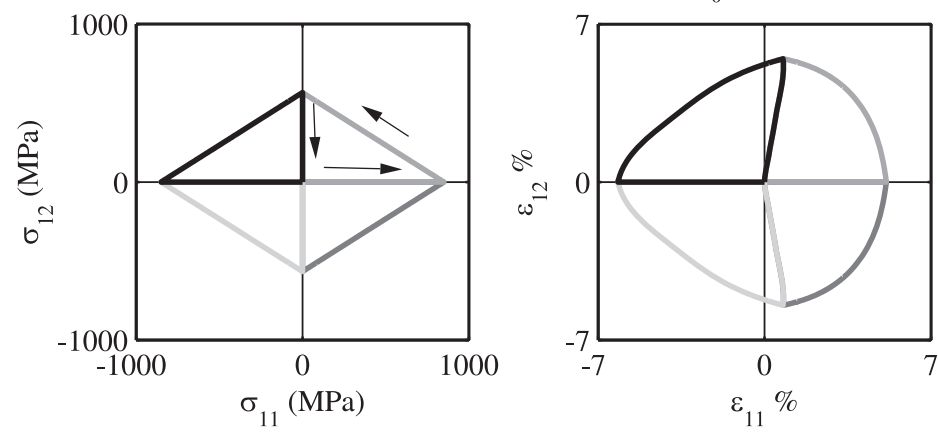

$\mathrm{a}=1.5 \mathrm{~b}=4.9 \mathrm{e}-5 \mathrm{c}=-0.6 \quad \mathrm{e}=<1 \quad 0 \quad 0>\theta_{0}=295$
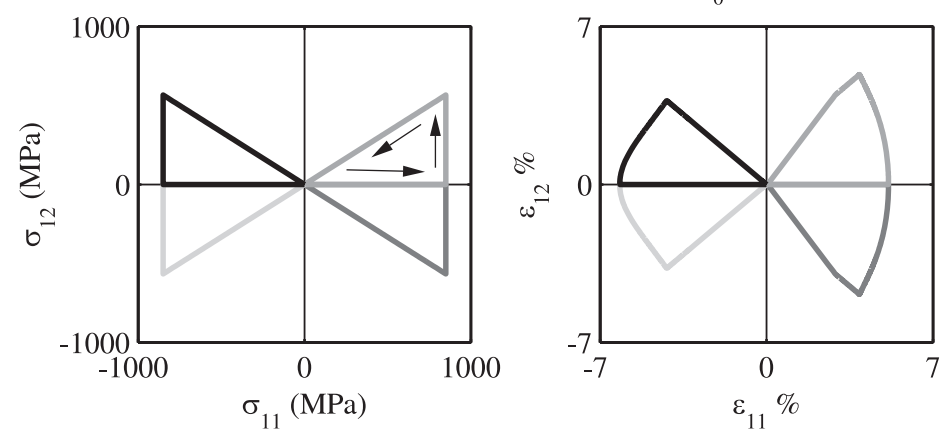

Figure 12. Applied stress and corresponding strain for material I for various paths. Each row shows the results of four symmetry-related paths.

The sets $P$ and $Y$ corresponding to these values are shown in figure 4 , and compared with the experiments to show a very good fit.

\subsection{Proportional loading}

For the material parameters chosen in the previous two sections, we simulate the response of the model for a few proportional loading experiments by imposing time-dependent proportional stress, and calculating the transformation strain, the total strain, volume fraction of the martensite and temperature are calculated. We keep the loading rate small enough so that the response is essentially quasi-static and choose the yield strength high enough to prevent any plastic deformation.

Figure 5 shows results of the uniaxial tensioncompression tests under adiabatic conditions for different initial temperatures. The evolution of the temperature and the volume fraction of martensite are also shown. The timescale is chosen such that the first half (time $0-0.5$ ) corresponds to ten- sion while the second half (time 0.5-1) corresponds to compression. All these temperatures are above the $A_{\mathrm{s}}$ temperature and the specimen is initially taken to be in the austenite state. The material deforms linearly as the loading begins. As the stress reaches a critical value, the transformation begins with an apparent yield in the stress-strain curve and proceeds to completion with increasing load and subsequently resumes its linear elastic response. Note that the temperature increases with transformation. As the load reaches its peak and starts to decrease, the reverse transformation takes place, fully recovering the strain and thus describing the superelastic response of the material. The compressive half-cycle is similar, though it requires a larger stress for the transformation and has a smaller transformation strain. This is consistent with the positive value of the parameter $a$. Further, the applied stress is not high enough to complete or even initiate the transformation at the higher temperatures.

These series of tests were confined to a temperature range where the material is superelastic. We refer the reader to [26] for a more complete study of the effect of initial temperature, 

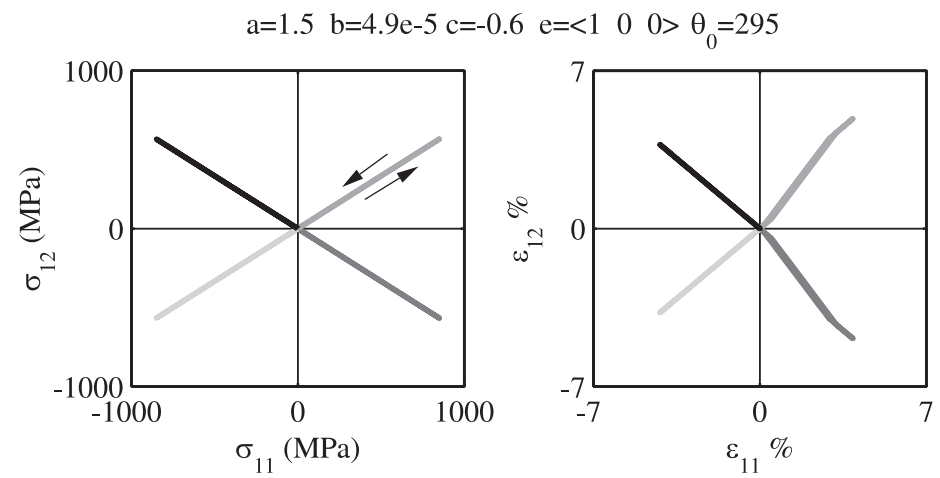

$a=1.5 \quad b=4.9 e-5 \quad c=-0.6 \quad e=<1 \quad 0 \quad 0>\theta_{0}=295$
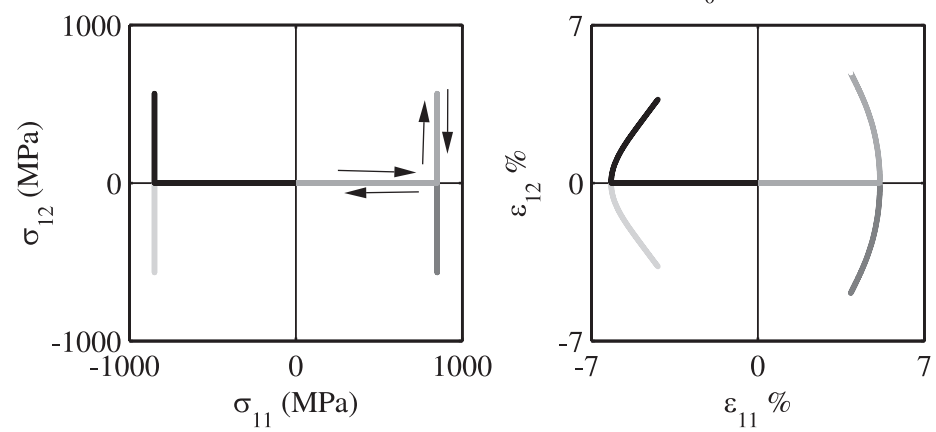

$a=1.5 \quad b=4.9 e-5 c=-0.6 \quad e=<1 \quad 0 \quad 0>\theta_{0}=295$
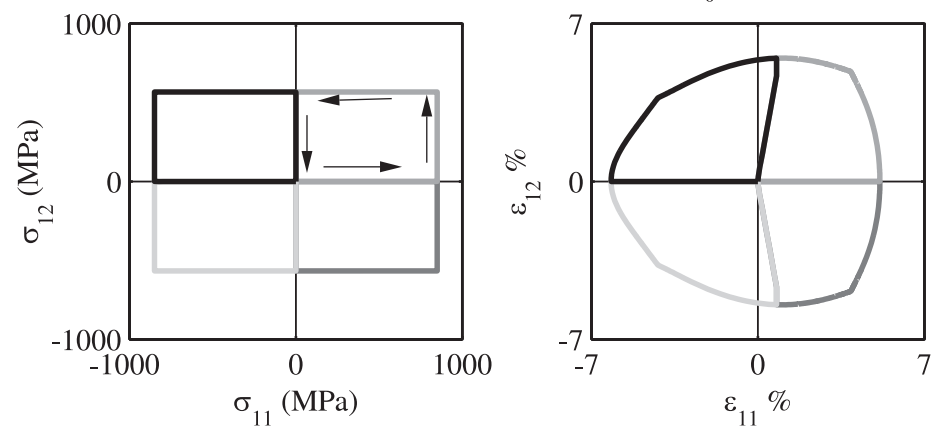

Figure 13. Applied stress and corresponding strain for material I for various paths. Each row shows the results of four symmetry-related paths.

loading rate and yield stress, as well as a demonstration of the shape-memory effect. Figure 6 shows the stressstrain response, growth of volume fraction of martensite and temperature as a function of time for pure shear tests for different initial temperatures.

Figure 7 shows results of an isothermal biaxial tensioncompression tests. The material is initially in austenite phase and subjected to a stress of the form

$$
\sigma=s(t)\left(\begin{array}{ccc}
\cos \phi & 0 & 0 \\
0 & \sin \phi & 0 \\
0 & 0 & 0
\end{array}\right)
$$

with $\phi$ held fixed. The temperature is held fixed at $220 \mathrm{~K}$ and the transformation strain, strain and martensite volume fraction are calculated as a function of time. This is repeated with various values of $\phi$ and the stress and strain paths are plotted variously in figure 7 .

Notice that strain begins evolving in the direction of the deviatoric part of the applied stress:

$$
\begin{aligned}
\sigma= & \frac{s(t)}{3} \\
& \times\left(\begin{array}{ccc}
2 \cos \phi-\sin \phi & 0 & 0 \\
0 & 2 \sin \phi-\cos \phi & 0 \\
0 & 0 & -\cos \phi-\sin \phi
\end{array}\right)
\end{aligned}
$$

till it hits the boundary of the set $P$. It then evolves along the boundary till it reaches the point with maximum projection along the deviatoric part of the applied stress. In other words, the strain response is not necessarily proportional. Consequently, a plot of the uniaxial stress component versus the uniaxial strain component can look quite complicated, as is also shown in figure 7.

\subsection{Non-proportional loading}

We now turn to non-proportional loading with the same parameters as above and under adiabatic conditions. We consider a combination of uniaxial tension and simple shear 

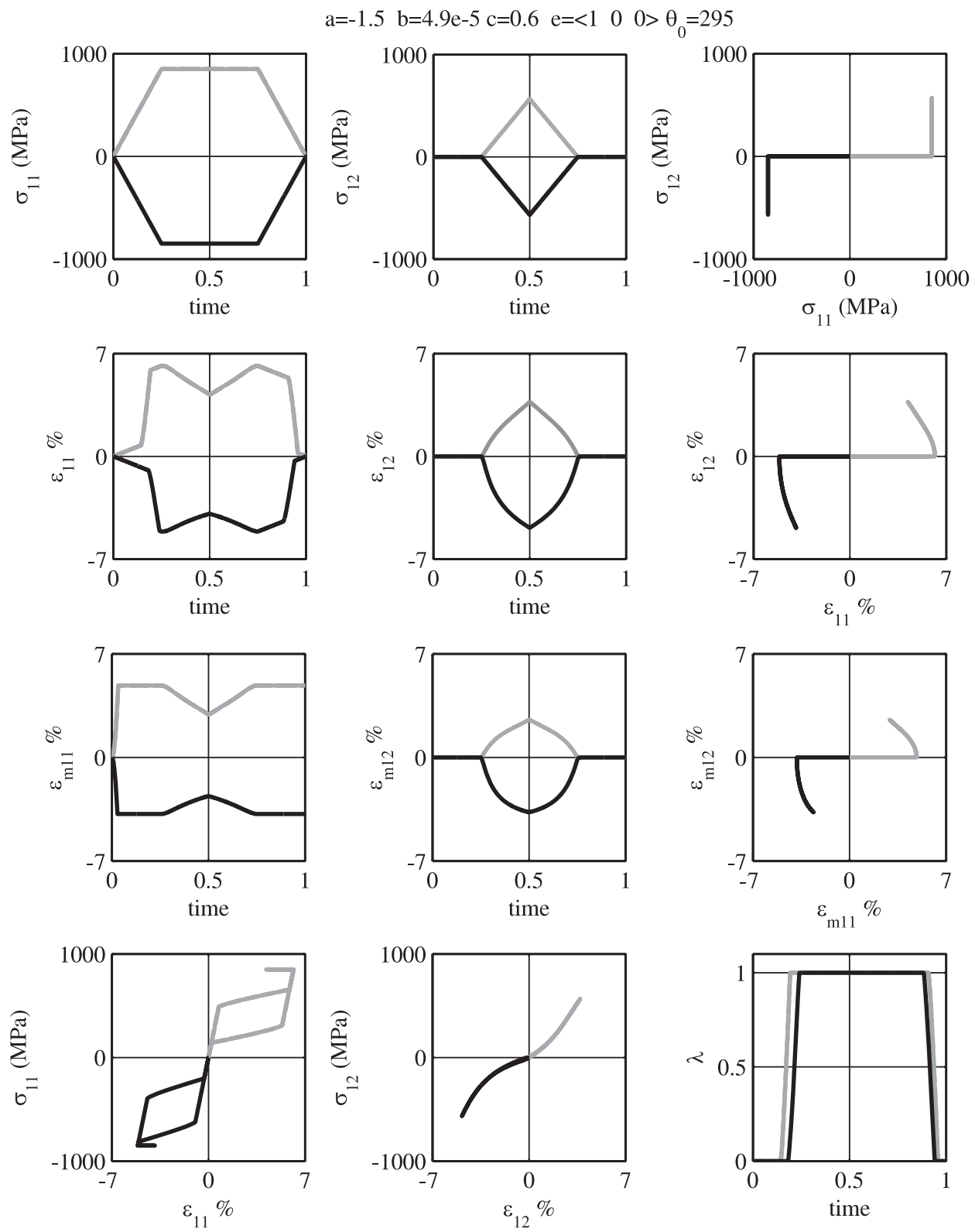

Figure 14. Stress-controlled behavior of the transversely isotropic model: material II.

mimicking the state of stress that a material point experiences in a tension-torsion experiment. As before we apply a timedependent stress and calculate the other variables according to our model.

Figure 8 shows the results of two stress paths. In the first, shown in dark, we apply an increasing tensile stress till it reaches $800 \mathrm{MPa}$, hold the stress fixed at this value while applying and then removing the shear stress, and finally unload the tensile stress. Note that the tensile component of the transformation strain $\varepsilon_{\mathrm{m} 11}$, and consequently the tensile component of the strain $\varepsilon_{11}$ decreases with increasing shear though the tensile stress is held fixed, but recovers with the removal of the shear. This reflects the shape of the set of transformation strains.

The light curves of figure 8 show the results for a second loading path. Here, the shear is applied first, held fixed while the tensile load is applied and removed, and finally the shear is unloaded. We see similar changes in the shear strain while the tensile stress is applied and removed.

Figure 9 shows similar stress paths with different signs of applied load. The phase transformation strain $\varepsilon_{\mathrm{m} 12} \rightarrow-\varepsilon_{\mathrm{m} 12}$ is symmetric and thus only the first and third quadrants of the phase transformation surface are necessary to predict the shape of the transformation surface in the $\varepsilon_{\mathrm{m}}$ space.

\section{Demonstration of the transversely isotropic model}

We now turn to the transversely isotropic material. We retain the parameters described by (45). 


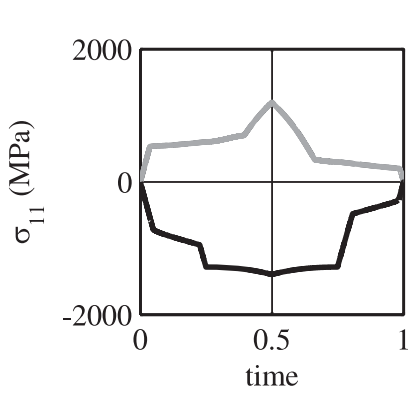

$a=-1.5 \quad b=4.9 e-5 \quad c=0.6 \quad e=<1 \quad 0 \quad 0>\theta_{0}=295$
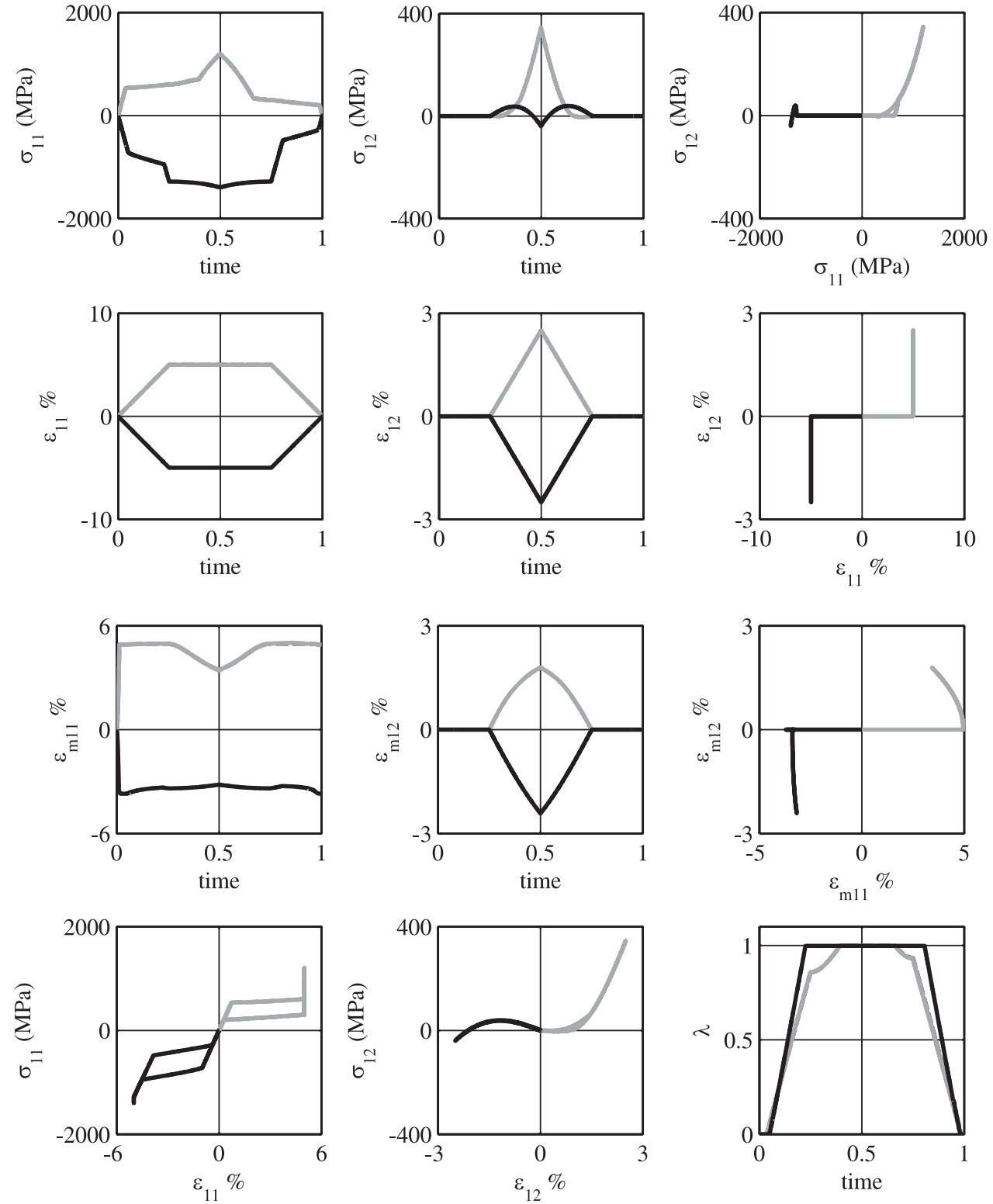

Figure 15. Strain-controlled behavior of the transversely isotropic model: material II.

\subsection{Set of effective transformation strains}

We consider the uniaxial or transversely isotropic material where the set of recoverable strains is described by (34). We select $e=\left\{\begin{array}{lll}1 & 0 & 0\end{array}\right\}$ to be the special direction of the texture without any loss of generality. The set of transformation strains is described by three parameters $a, b$ and $c$. The parameter $b$ scales the set and the parameter while the parameters $a$ and $c$ describe the asymmetry and anisotropy.

Figure 10 shows the section of the set $P$ with the plane

$$
\epsilon_{\mathrm{m}}=\left(\begin{array}{ccc}
\epsilon_{\mathrm{m} 11} & 0 & 0 \\
0 & \epsilon_{\mathrm{m} 22} & 0 \\
0 & 0 & -\epsilon_{\mathrm{m} 11}-\epsilon_{\mathrm{m} 22}
\end{array}\right) .
$$

Each figure on the top row labeled (a) shows four different values of $a$ as $c$ changes from negative to positive, while each figure on the bottom row labeled (b) shows four different values of $c$ as $a$ changes from negative to positive. The case $c=0$ on the middle of the top row corresponds to the isotropic situation.

Figure 11 shows the section of the set $P$ with the plane

$$
\epsilon_{\mathrm{m}}=\left(\begin{array}{ccc}
\epsilon_{\mathrm{m} 11} & \epsilon_{\mathrm{m} 12} & 0 \\
\epsilon_{\mathrm{m} 12} & 0 & 0 \\
0 & 0 & -\epsilon_{\mathrm{m} 11}
\end{array}\right)
$$

Each figure on the top row labeled (a) shows four different values of $a$ as $c$ changes from negative to positive, while each figure on the bottom row labeled (b) shows four different values of $c$ as $a$ changes from negative to positive. The case $c=0$ on the middle of the top row corresponds to the isotropic situation. 


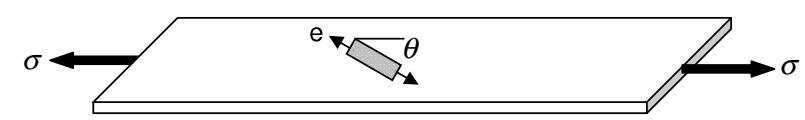

Figure 16. Rolled sheet under tensile stress.

\subsection{Stress and strain controlled tests}

We describe the response of the transversely isotropic material in various stress and strain controlled tests using the following set of parameters:

- Material I: $a=1.5, b=0.000049$ and $c=-0.6$,

- Material II: $a=-1.5, b=0.000049$ and $c=0.6$.

All other parameters are fixed at the values described earlier.

Figures 12 and 13 show the stress-strain response of material 1 for six different stress-controlled loading paths in the $\sigma_{11}-\sigma_{12}$ space. These paths were inspired by the experiments of Sittner et al [28], though they were conducted on a different copper-based material.

Figure 14 shows the response of the model under applied non-proportional tension-shear stress for material II. The initial temperature is $295 \mathrm{~K}$ and the situation is assumed to be adiabatic.

Figure 15 shows the response of the model under nominally strain-controlled tension-shear tests. In these simulations $\epsilon_{11}$ and $\epsilon_{12}$ are prescribed to given time-dependent values, $\epsilon_{22}$ is prescribed to be zero and all lateral stress components $\sigma_{3 i}$ are set to zero. We do so because this is the most common condition in tension-torsion tests, and also because it demonstrates the ability of using the model under complex conditions. Notice by comparison to figure 14 that the stress-strain curves can look quite different under stresscontrolled and strain-controlled situations even though the imposed stress and strain histories look similar.

\subsection{Rolling texture}

We conclude by demonstrating that our model can be fit to one observed rolling texture. Inoue et al [10] have investigated planar anisotropy of shape-memory strain in polycrystalline NiTi alloy sheets by conducting uniaxial stressstrain measurements on small specimens extracted at different orientations from a rolled sheet as shown in figure 16. Figure 17 shows how our model is capable of reproducing their experimental observations. The parameters used for this fitting are similar to those listed in section 3.1 along with the following:

$$
a=-1.5, \quad b=0.000063, \quad c=0.3 .
$$

\section{Conclusion and discussion}

A micromechanics-inspired constitutive model for polycrystalline shape-memory alloys in three dimensions is presented. The model is a generalization of the one-dimensional model presented earlier [26] and remains applicable in a wide range of temperatures and strain rates. It is able to describe satisfactorily the stress-strain response for complex proportional and

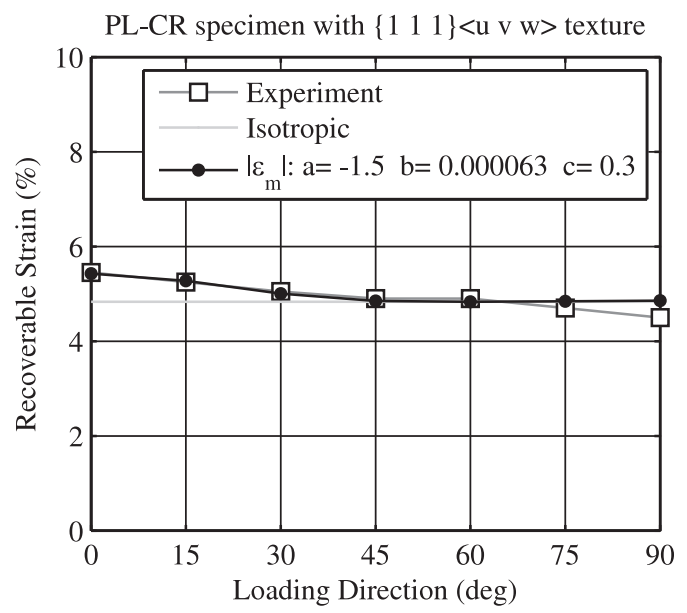

Figure 17. Analytical model versus experiment, PL-CR ( $\left\{\begin{array}{lll}1 & 1 & 1\end{array}\right\}\langle u v w\rangle$ texture).

non-proportional loading patterns and can simulate the effect of texture pf a polycrystal of shape-memory alloy.

While the model seeks to incorporate the lessons of various micromechanical analysis, it does rely on a number of modeling hypotheses. In particular, one that is significant is the assumption that the austenite and the martensite have the same elastic moduli. This is of course not the case in reality. Consequently, the elastic modulus $C$ is a function of volume fraction $\lambda$, i.e. $C=C(\lambda)$ in equation (7). This changes the formula for the driving force $d_{\lambda}$ in equation (11) and makes some of the calculations more complicated. These changes can easily be implemented. However, the issue of elastic heterogeneity is deeper. There is a self-energy generated by the elastic heterogeneity and this can be significant. Consequently, one needs to add a term $G\left(\varepsilon, \lambda, \varepsilon_{\mathrm{m}}\right)$ to the energy in equation (7) to represent the self-energy of the optimal microstructure with total strain $\varepsilon$, volume fraction $\lambda$ and effective transformation strain $\varepsilon_{\mathrm{m}}$. However, a satisfactory constitutive model for this $G$ remains unclear at the moment. This remains a topic of current research, and an open issue for future work.

\section{Acknowledgments}

We gratefully appreciate the partial financial support of the Army Research Office through the MURI grant DAAD 19-010517 and the Office of Naval Research through N00014-05-10202 .

\section{References}

[1] Auricchio F, Taylor R L and Lubliner J 1997 Shape-memory alloys: macromodelling and numerical simulations of the superelastic behavior Comput. Methods Appl. Mech. Eng. 146 281-312

[2] Auricchio F and Petrini L 2004 A three-dimensional model describing stress-temperature induced solid phase transformations: solution algorithm and boundary value problems Int. J. Numer. Methods Eng. 61 807-36 
[3] Bhattacharya K 2003 Microstructure of Martensite (Oxford: Oxford University Press)

[4] Bhattacharya K and Kohn R V 1997 Energy minimization and the recoverable strains of polycrystalline shape-memory alloys Arch. Ration. Mech. Anal. 139 99-180

[5] Bhattacharya K and Schlömerkemper A 2004 Transformation yield surface of shape-memory alloys J. Physique IV 115 $155-62$

[6] Bhattacharyya A, Lagoudas D C, Wang Y and Kinra V K 1995 On the role of thermoelectric heat transfer in the design of SMA actuators: theoretical modelling and experiments Smart Mater. Struct. 4 252-63

[7] Boyd J G and Lagoudas D C 1996 A thermodynamic constitutive model for shape memory materials: 1 . The monolithic shape memory alloy Int. J. Plast. 12 843-73

[8] Brocca M, Brinson L C and Bazant Z 2002 Three-dimensional constitutive model for shape memory alloys based on microplane J. Mech. Phys. Solids 50 1051-77

[9] Burkart M W and Read T A 1953 Trans. TMS-AIME 197 1516-24

[10] Inoue H, Miwa N and Inakazu N 1996 Texture and shape memory strain in TiNi alloy sheets Acta Mater. 44 4825-34

[11] Jung Y J, Papadopoulos P and Ritchie R O 2004 Constitutive modelling and numerical simulation of multivariant phase transformation in superelastic shape-memory alloys Int. $J$. Numer. Methods Eng. 60 429-60

[12] Lexcellent C and Blanc P 2004 Phase transformation yield surface determination for some shape memory alloys Acta Mater. 52 2317-24

[13] Lexcellent C, Goo B C, Sun Q P and Bernardini J 1996 Characterization, thermomechanical behaviour and micromechanical-based constitutive model of shape-memory $\mathrm{Cu}-\mathrm{Zn}-\mathrm{Al}$ single crystals Acta Mater. 44 3773-80

[14] Lexcellent C, Vivet A, Bouvet C, Calloch S and Blanc P 2002 Experimental and numerical determinations of the initial surface of phase transformation under biaxial polycrystalline shape-memory alloys J. Mech. Phys. Solids 50 2717-35

[15] Lu Z K and Weng G J 1997 Martensitic transformation and stress-strain relations of shape-memory alloys J. Mech. Phys. Solids 451905

[16] Marketz F and Fischer F D 1996 Modelling the mechanical behavior of shape memory alloys under variant coalescence Comput. Mater. Sci. 5 210-26

[17] McNaney J M, Imbeni V, Jung Y, Papadopoulos P and Ritchie R O 2003 An experimental study of the superelastic effect in a shape-memory Nitinol alloy under biaxial loading Mech. Mater. 35 969-86

[18] Naito H, Matsuzaki Y and Ikeda T 2004 A unified constitutive model of phase transformations and rearrangements of shape memory alloy wires subjected to quasistatic load Smart Mater. Struct. 13 535-43

[19] Nemat-Nasser S, Choi J Y, Guo W G, Isaacs J B and Taya M 2005 High strain-rate, small strain response of a NiTi shape-memory alloy J. Eng. Mater. Tech. 127 83-9
[20] Niclaeys C, Ben Zineb T, Arbab-Chirani S and Patoor E 2002 Determination of the interaction energy in the martensitic state Int. J. Plast. 18 1619-47

[21] Otsuka K and Wayman C M 1998 Shape Memory Materials (Cambridge: Cambridge University Press)

[22] Paiva A, Savi M A, Braga A M B and Pacheco P M C L 2005 A constitutive model for shape memory alloys considering tensile-compressive asymmetry and plasticity Int. J. Solids. Struct. 42 3439-57

[23] Purohit P K 2002 Dynamics of phase transformations in strings, beams and atomic chains $P h D$ Thesis California Institute of Technology

[24] Qidwai M A and Lagoudas D C 2000 Numerical implementation of a shape memory alloy thermomechanical constitutive model using return mapping algorithms Int. $J$. Numer. Methods Eng. 47 1123-68

[25] Sadjadpour A 2006 Micromechanics-inspired three-dimensional constitutive model for the thermomechanical response of shape-memory alloys Doctoral Dissertation California Institute of Technology

[26] Sadjadpour A and Bhattacharya K 2007 A micromechanics inspired constitutive model for shape-memory alloys: the one-dimensional case Smart Mater. Struct. 16 S51-62

[27] Shu Y C and Bhattacharyya K 1998 The influence of texture on the shape-memory effect in polycrystal Acta Mater. 46 5457-73

[28] Sittner P, Hara Y and Tokuda M 1995 Experimental study on the thermoelastic martensitic transformation in shape memory alloy polycrystal induced by combined external forces Metall. Mater. Trans. 26 2923-35

[29] Stoijov V and Bhattacharyya A 2002 A theoretical framework of one-dimensional sharp phase fronts in shape memory alloys Acta Mater. 50 4939-52

[30] Sun Q P and Hwang K C 1993 Micromechanics modeling for the constitutive behavior of polycrystalline shape-memory alloys: 1. Derivation of general relations J. Mech. Phys. Solids 41 1-17

Sun Q P and Hwang K C 1993 Micromechanics modeling for the constitutive behavior of polycrystalline shape-memory alloys: 2. Study of individual phenomena J. Mech. Phys. Solids 41 19-33

[31] Thamburaja P and Anand L 2001 Polycrystalline shape-memory materials: effect of crystallographic texture J. Mech. Phys. Solids 49 709-37

[32] Wang Y H and Fang D N 2003 A three-dimensional constitutive model for shape memory alloys Int. J. Nonlinear Sci. Numer. Simul. 4 81-7

[33] Zaki W and Moumni Z 2005 Thermomechanical modelization of shape-memory alloys: generalization of the Moumni-Son law J. Physique IV 124 237-42

[34] Zhu J J, Huang N G, Liew K M and Zhu Z H 2002 A thermodynamic constitutive model for stress induced phase transformation in shape memory alloys Int. J. Solids Struct. $39741-63$ 